\title{
MicroRNA-215 acts as a tumor suppressor in breast cancer by targeting AKT serine/threonine kinase 1
}

\author{
JIAN YAO, PING ZHANG, JIN LI and WEI XU \\ Department of Integrated Traditional Chinese and Western Medicine, \\ First Affiliated Hospital of Harbin Medical University, Harbin, Heilongjiang 150001, P.R. China
}

Received February 7, 2016; Accepted February 7, 2017

DOI: $10.3892 / \mathrm{ol} .2017 .6200$

\begin{abstract}
There are accumulating reports that microRNAs are dysregulated in a number of human cancer types, and that they may function as tumor suppressors or oncogenes in tumorigenesis and tumor development. microRNA-215 (miR-215) has been identified as a tumor suppressor in epithelial ovarian, pancreatic, non-small cell lung and colon cancer, whereas it may act as an oncogene in gastric and cervical cancer. The role of miR-215 in breast cancer carcinogenesis and progression has yet to be elucidated. In the present study, the expression level of miR-215 was determined in breast cancer tissues and cell lines using the reverse transcription-quantitative polymerase chain reaction. The effects of miR-215 overexpression on proliferation and the invasive capacity of breast cancer cells were assessed using MTT and cell invasion assays. The results revealed that miR-215 was significantly downregulated in breast cancer tissues and cell lines. Restoration of miR-215 expression inhibited the proliferation and invasion of breast cancer cells. The underlying molecular mechanism for the suppression of proliferation and invasion by miR-215 was investigated. AKT serine/threonine kinase 1 (AKT1) was validated as a novel direct target of miR-215, and the effect of AKT1 small interfering RNA mimicked the effect of miR-215 overexpression in breast cancer cells. These results indicated that miR-215 acted as a tumor suppressor, and that its downregulation in tumor tissues may contribute to the carcinogenesis and progression of breast cancer, indicating that miR-215 may be a novel therapeutic target for the treatment of breast cancer.
\end{abstract}

Correspondence to: Professor Wei Xu, Department of Integrated Traditional Chinese and Western Medicine, First Affiliated Hospital of Harbin Medical University, 23 You Zheng Street, Harbin, Heilongjiang 150001, P.R. China

E-mail: xw2015great@163.com

Key words: breast cancer, microRNA-215, tumor suppressor, AKT serine/threonine kinase, targeted therapy

\section{Introduction}

Breast cancer is the leading cause of cancer-associated mortality for the female population worldwide; there were an estimated 234,190 novel cases and 40,730 mortalities in 2015 in the USA $(1,2)$. Treatment of breast cancer typically includes surgery followed by adjuvant chemotherapy, radiotherapy or hormone therapy (3). There has been a significant effort to improve the prognosis of patients with breast cancer; however, breast cancer remains a highly prevalent and lethal malignancy $(4,5)$. Local recurrence and distant metastasis are the main reasons for the poor prognosis associated with breast cancer (6). It was previously reported that between 30 and $75 \%$ of patients with breast cancer developed disease recurrence or metastasis following surgery and adjuvant treatment (7). The local recurrence or metastasis of breast cancer is the leading cause for the deterioration of breast cancer, and is responsible for $>90 \%$ of breast cancer-associated mortalities (8). Therefore, improving the understanding of the molecular mechanisms of breast cancer recurrence and metastasis is particularly important.

MicroRNAs (miRNAs), small non-coding RNAs of 19-24 nucleotides, may be critical regulators of cancer pathogenesis and progression (9). miRNAs negatively regulate the expression of specific mRNA targets by directly binding miRNA recognition elements, typically in the $3^{\prime}$ untranslated region (3'UTR) of target mRNAs, resulting in the inhibition of translation and/or degradation of the target mRNA, thus downregulating the expression of the associated protein products $(10,11)$. Each type of miRNA may modulate the expression of numerous mRNAs simultaneously; therefore, miRNAs serve critical roles in biological functions, including cell proliferation, cell cycle progression, migration, invasion, metastasis, apoptosis, metabolism and the immune response (12-18). Accumulating studies have indicated that there are associations between miRNA expression and the clinical features of patients with cancer, including tumor size, tumor-node-metastasis (TNM) stage, development, survival time, disease recurrence and metastasis (19-22). miRNAs have been reported to be frequently dysregulated in various types of human cancer, and may function as tumor suppressors or oncogenes in tumorigenesis and tumor development (23). miRNAs are therefore regarded as attractive targets for the development of improved cancer therapies. 
In the present study, it was demonstrated that the expression levels of miR-215 were lower in breast cancer tissues and cell lines. In addition, enforced miR-215 expression inhibited the proliferation and invasion of breast cancer cells. AKT threonine serine/kinase 1 (AKT1) was identified as a novel direct target of miR-215 in breast cancer. On the basis of these results, miR-215 may be suitable for development as a therapeutic target in the treatment of breast cancer.

\section{Materials and methods}

Clinical samples and cell lines. Breast cancer and matched adjacent non-tumor breast tissues were obtained from 56 patients who underwent surgery at the First Affiliated Hospital of Harbin Medical University (Harbin, China) between September 2013 and February 2014. None of the patients had received chemotherapy, radiotherapy or adjuvant hormonal therapy prior to surgery. All samples were snap frozen in liquid nitrogen and stored at $-80^{\circ} \mathrm{C}$. The present study was approved by the Ethics Committee of the First Affiliated Hospital of Harbin Medical University. Written informed consent for research purposes was obtained from each patient.

Human breast cancer cells (MCF-7A, MDA-MB-231, MDA-MB-453, BT-474 and SK-BR-3) and HEK293T cells were obtained from the American Type Culture Collection (Manassas, VA, USA). MCF-10A, normal mammary epithelial cells, were purchased from the Cell Type Culture Collection of the Chinese Academy of Sciences (Shanghai, China). Cells were maintained in Dulbecco's modified Eagle's medium (DMEM) supplemented with 10\% fetal bovine serum (FBS), $100 \mathrm{U} / \mathrm{ml}$ penicillin and $100 \mu \mathrm{g} / \mathrm{ml}$ streptomycin (Gibco; Thermo Fisher Scientific, Inc., Waltham, MA, USA) at $37^{\circ} \mathrm{C}$ in a humidified atmosphere containing $5 \% \mathrm{CO}_{2}$.

miRNA and small interfering RNA (siRNA) transfection. An miR-215 mimic and an miRNA negative control (NC) were synthesized by Shanghai GenePharma Co., Ltd. (Shanghai, China). AKT1 siRNA and scrambled siRNA were purchased from Guangzhou RiboBio Co., Ltd. (Guangzhou, China). The breast cancer cells were seeded into 6-well plates and grown to $60 \%$ confluence. They were transfected with miRNA or siRNA using Lipofectamine 2000 (Invitrogen; Thermo Fisher Scientific, Inc.), according to the manufacturer's protocol.

Reverse transcription-quantitative polymerase chain reaction (RT-qPCR). Total RNA was extracted from tissues and cells with TRIzol reagent (Invitrogen; Thermo Fisher Scientific, Inc.), according to the manufacturer's protocol. For detecting miR-215 expression, total RNA was reverse-transcribed into complementary DNA with a PrimeScript reverse transcription reagent kit (Takara Bio, Inc., Otsu, Japan). qPCR was performed with a SYBR Premix Ex Taq ${ }^{\mathrm{TM}}$ II kit (Takara Bio, Inc.) on an Applied Biosystems 7500 Sequence Detection system (Thermo Fisher Scientific, Inc.).

The reaction system contained $10 \mu \mathrm{l}$ SYBR Premix Ex Taq II, $2 \mu \mathrm{l}$ cDNA (200 ng), $0.8 \mu \mathrm{l}$ forward primer, $0.8 \mu \mathrm{l}$ reverse primer, $0.4 \mu \mathrm{l}$ ROX Reference Dye and $6 \mu \mathrm{l}$ ddH2O. The amplification was performed with cycling conditions as follows: $5 \mathrm{~min}$ at $95^{\circ} \mathrm{C}$, followed by 40 cycles of $95^{\circ} \mathrm{C}$ for $30 \mathrm{sec}$ and $65^{\circ} \mathrm{C}$ for $45 \mathrm{sec}$. Relative expression levels of miR-215 were evaluated using the $2^{-\Delta \Delta \mathrm{Cq}}$ method (24). The primer sequences used for qPCR were: miR-215 forward, 5'-ACACTCCAGCTGGGATGACCTATGAATTG-3' and reverse, 5'-GTGCAGGGTCCGAGGT-3'; U6 snRNA forward, 5'-CTCGCTTCGGCAGCACATATACT-3' and reverse, 5'-ACGCTTCACGAATTTGCGTGTC-3'. All experiments were run in triplicate.

MTT assay. An MTT assay was performed to detect the rate of cell proliferation. Breast cancer cells were seeded in a 96-well plate, at a density of $3 \times 10^{3}$ cells per well, following transfection with miR-215 mimic or siRNA. The extent of proliferation was then assessed at 24, 48, 72 and $96 \mathrm{~h}$ post-transfection. A $20 \mu 1$ volume of MTT solution $(5 \mathrm{mg} / \mathrm{ml}$; Sigma-Aldrich; Merck KGaA, Darmstadt, Germany) was added to each well prior to incubation at $37^{\circ} \mathrm{C}$ for $4 \mathrm{~h}$. The supernatant was removed and cells were resuspended in $150 \mu \mathrm{l}$ dimethylsulfoxide. The absorbance of the cells at $490 \mathrm{~nm}$ was detected using a microplate reader. All experiments were performed in triplicate.

Cell invasion assay. A Transwell chamber with $8 \mu \mathrm{m}$ pores (BD Biosciences, San Jose, CA, USA) was used to evaluate cell invasive capacity. The filter of the top chamber was pre-coated with Matrigel (BD Biosciences). Briefly, 4x10 miR-215 mimic- or siRNA-transfected cells were suspended in $200 \mu \mathrm{l}$ FBS-free DMEM, and added to the upper chamber. The lower chambers were filled with $500 \mu 1$ DMEM supplemented with $20 \%$ FBS as a chemoattractant. At $48 \mathrm{~h}$ after incubation, the noninvading cells that remained on the upper surface of the Transwell chamber were removed with a cotton swab. The invading cells on the lower surface were fixed with $100 \%$ methanol and stained with $0.1 \%$ crystal violet. Cells that had invaded to the lower surface were counted using a light microscope in five randomly selected fields.

Bioinformatics analysis. TargetScan online software (www. targetscan.org/) and miRanda (www.microrna.org) were used for miR-215 target gene prediction. From the potential targets, AKT1 was selected as it has previously been demonstrated to contribute to breast cancer progression (25).

Western blot analysis. At $72 \mathrm{~h}$ after transduction with the miR-215 mimic or siRNA, total protein was extracted from cells using a Total Protein Extraction kit (Nanjing KeyGen Biotech Co., Ltd., Nanjing, China) and its concentration was determined using a Pierce bicinchoninic acid protein quantitation kit (Thermo Fisher Scientific, Inc.), according to the manufacturer's protocol. Equal amounts of protein $(30 \mu \mathrm{g})$ were separated using SDS-PAGE (10\% gel) and gels were electroblotted onto polyvinylidene fluoride membranes (Merck $\mathrm{KGaA})$. The membranes were blocked with $5 \%$ skimmed milk in TBS containing $0.1 \%$ Tween-20, and then incubated with the following primary antibodies: Mouse anti-human AKT1 monoclonal antibody (ab54752, 1:1,000; Abcam, Cambridge, UK) and mouse anti-human $\beta$-actin monoclonal antibody (ab8226, 1:1,000; Abcam). Following overnight incubation at $4^{\circ} \mathrm{C}$, the membranes were probed with a goat anti-mouse secondary antibody (ab6789, 1:5,000; Abcam) for $1 \mathrm{~h}$ at room 

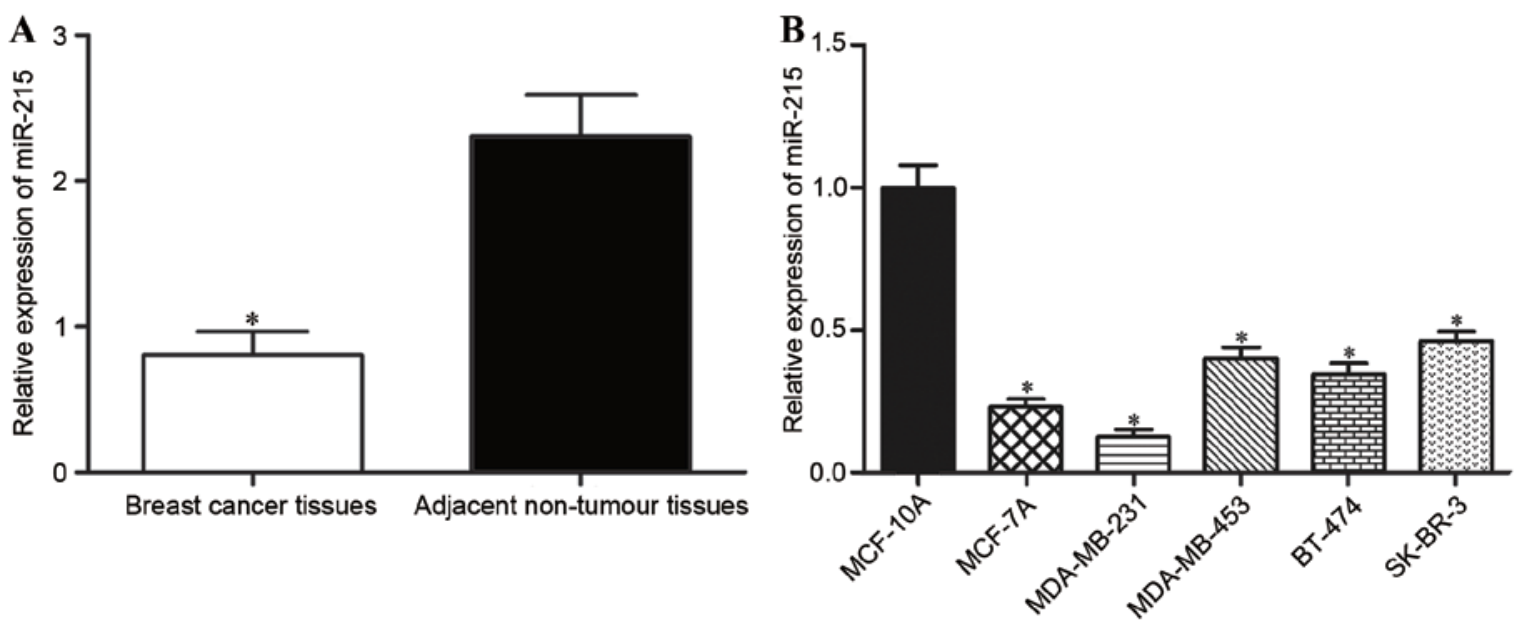

Figure 1. miR-215 was downregulated in breast cancer tissues and cell lines. (A) Expression levels of miR-215 were significantly decreased in the breast cancer tissues compared with matched adjacent non-tumor breast tissues. Data are presented as the mean \pm standard deviation. ${ }^{*} \mathrm{P}<0.05$ vs. adjacent non-tumor breast tissues. (B) Relative expression of miR-215 was lower in all breast cancer cell lines examined compared with MCF-10A. "P $<0.05$ compared with MCF-10A. miR-215, microRNA-215.

temperature. The bands were visualized using Electrogenerated chemiluminescence (GE Healthcare Life Sciences, Chalfont, UK). $\beta$-Actin was used as a loading control for protein level normalization.

Luciferase reporter assay. The luciferase reporter vectors, pGL3-AKT1-3'UTR wild-type 1 (Wt1), 2 (Wt2), mutant 1 (Mut1) and mutant 2 (Mut2), were synthesized by Shanghai GenePharma Co., Ltd. For the luciferase reporter assay, breast cancer cells were cultured in 24-well plates and transfected with luciferase reporter vectors, along with the miR-215 mimic or NC, using Lipofectamine 2000. At $48 \mathrm{~h}$ after transfection, luciferase activities were measured with the Dual-Luciferase Reporter Assay system (Promega Corporation, Madison, WI, USA) according to the manufacturer's protocol. Firefly luciferase activity was normalized to Renilla luciferase activity.

Statistical analysis. Data were presented as mean \pm standard deviation. Statistical analysis was performed using Student's t-tests or one-way analysis of variance plus multiple comparisons using SPSS 16.0 software (SPSS, Inc., Chicago, IL, USA). SNK was used to compare between two groups in multiple groups. For all analyses, $\mathrm{P}<0.05$ was considered to indicate a statistically significant difference.

\section{Results}

miR-215 is downregulated in breast cancer tissues and cell lines. To evaluate the role of miR-215 in breast cancer, RT-qPCR was performed to measure the expression levels of miR-215 in breast cancer tissues and matched adjacent non-tumor breast tissues. The expression of miR-215 in breast cancer tissues was significantly decreased compared with in matched adjacent non-tumor breast tissues ( $\mathrm{P}<0.05$; Fig. 1A).

For further characterization of miR-215 expression in breast cancer, its expression in a normal mammary epithelial cell line (MCF-10A) and breast cancer cell lines (MCF-7A, MDA-MB-231, MDA-MB-453, BT-474 and SK-BR-3) was also quantified. RT-qPCR analysis demonstrated that miR-215 expression was significantly decreased in all the breast cancer cell lines compared with MCF-10A ( $\mathrm{P}<0.05$; Fig. 1B). The lowest level of miR-215 expression was in MCF-7A and MDA-MB-231 cells, which were selected for use in further experiments.

Effects of miR-215 on the proliferation and invasion of breast cancer cells. Subsequent to transfecting MCF-7A and MDA-MB-231 cells with the miR-215 mimic or NC, the expression level of miR-215 was measured using RT-qPCR. Transfection with the miR-215 mimic led to a significant increase in its expression in MCF-7A and MDA-MB-231 cells $(\mathrm{P}<0.05$; Fig. 2A).

An MTT assay was performed to evaluate the effect of miR-215 on the proliferation of MCF-7A and MDA-MB-231 cells. MCF-7A and MDA-MB-231 cells transfected with the miR-215 mimic proliferated at a decreased rate compared with the cells transfected with $\mathrm{NC}(\mathrm{P}<0.05$; Fig. 2B).

An invasion assay was performed to investigate the effect of miR-215 on the invasive capacity of breast cancer cells. Following transfection with the miR-215 mimic, the invasive capacity of MCF-7A and MDA-MB-231 cells was significantly decreased compared with NC-transfected cells (Fig. 2C). Taken together, the data indicated that miR-215 inhibited the proliferation and invasion of breast cancer cells.

Overexpression of miR-215 decreases the level of AKT1 protein. In order to investigate the underlying molecular mechanism of miR-215 in the progression of breast cancer, TargetScan and miRanda were used to screen for target genes of miR-215. From the potential targets, AKT1 was selected as it had previously been demonstrated to contribute to breast cancer progression. As illustrated in Fig. 3A, the 3'UTR of AKT1 contains two predicted binding sites for miR-215.

The effect of miR-215 overexpression on AKT1 protein expression in MCF-7A and MDA-MB-231 cells was evaluated using western blot analysis. AKT1 was significantly downregulated in miR-215 mimic-transfected MCF-7A and 

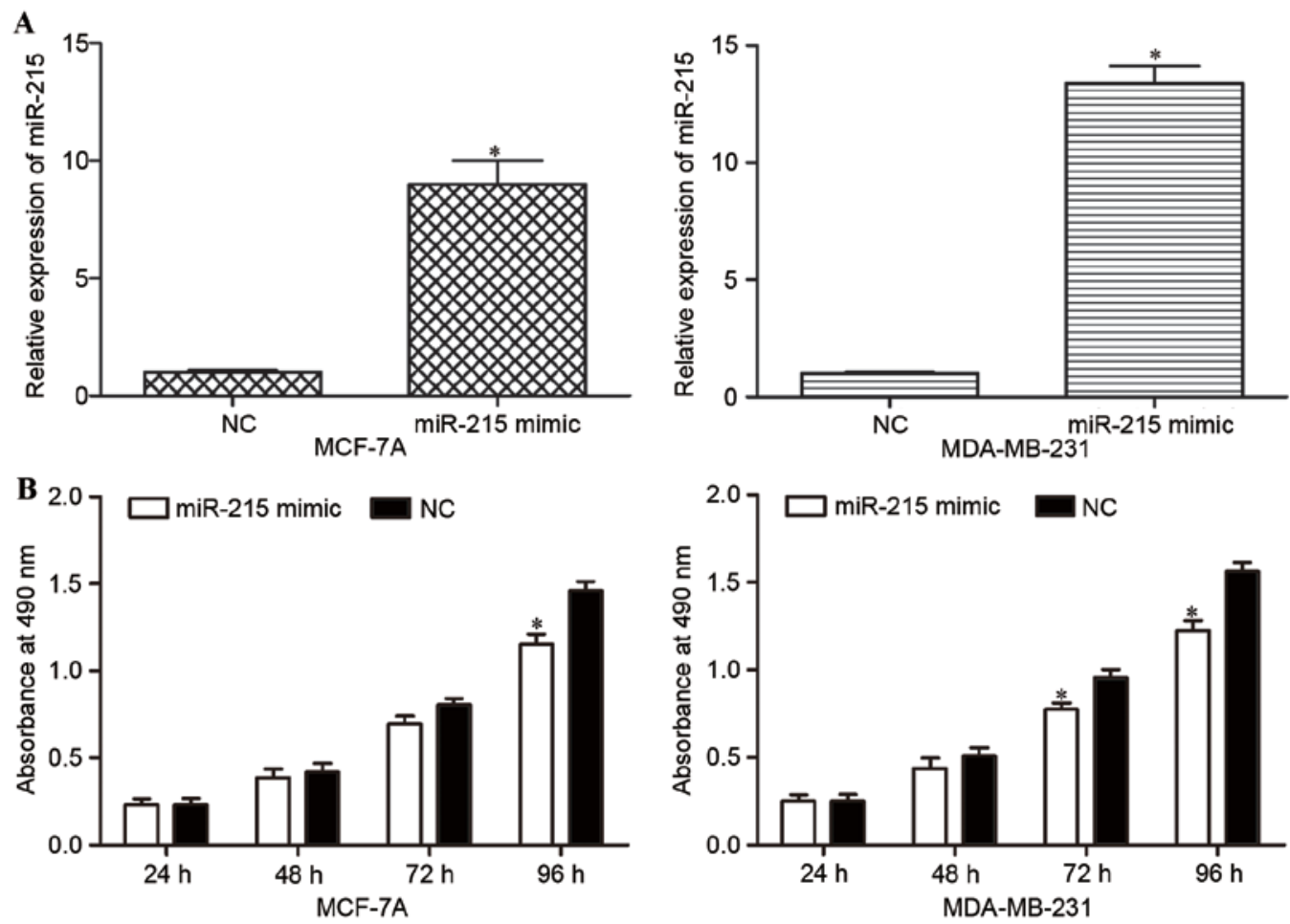

C
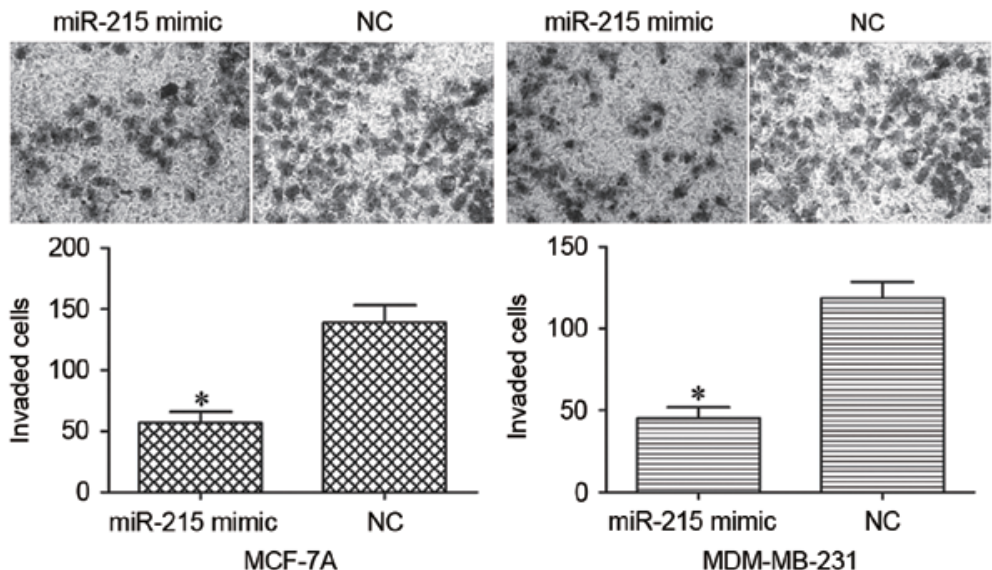

Figure 2. Effects of miR-215 overexpression on the proliferation and invasion of breast cancer cells. (A) Overexpression of miR-215 was simulated by transfecting miR-215 mimic into MCF-7A and MDA-MB-231 cells. miR-215 expression was measured using the reverse transcription-quantitative polymerase chain reaction $48 \mathrm{~h}$ after transfection. (B) Cell proliferation was determined using an MTT assay. MCF-7A and MDA-MB-231 cells transfected with miR-215 mimic exhibited a decreased rate of proliferation compared with cells transfected with NC. (C) miR-215 overexpression decreased the invasive capacity of MCF-7A and MDA-MB-231 cells compared with NC groups. "P<0.05 compared with NC-transfected cells. mirR-215, microRNA-215; NC, negative control.

A

Predicted binding site 1 AKT1 3' UTR Wt 1 5'

miR-215

AKT1 3' UTR mut 1 5'

Predicted binding site 2 AKT1 3' UTR Wt 1

miR-215

AKT1 3' UTR mut 2 5'

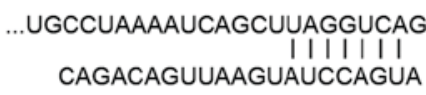

..GCCUGCCCAGCUACUAUCCAGUG
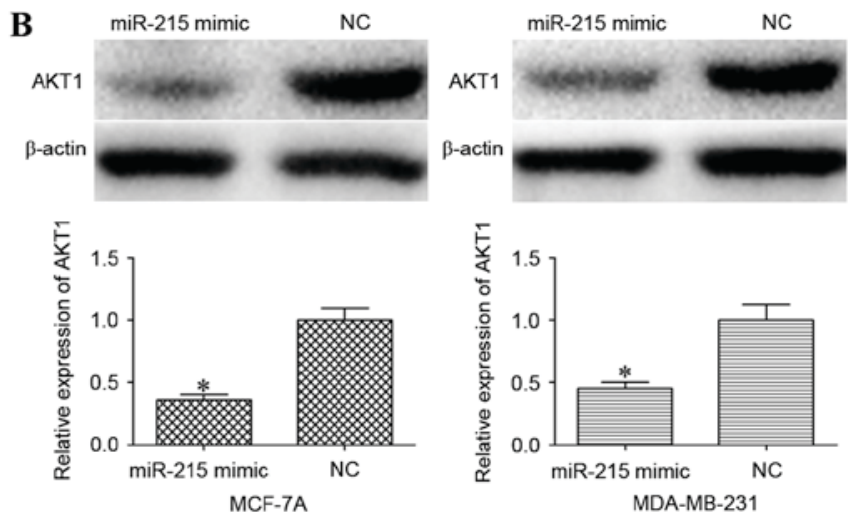

Figure 3. miR-215 overexpression decreased AKT1 expression in breast cancer cells. (A) miR-215-binding sites in the 3'UTR of AKT1. (B) The miR-215 mimic inhibited the expression of AKT1 at the protein level in MCF-7A and MDA-MB-231 cells. "P<0.05 compared with NC-transfected cells. miR-215, microRNA-215; AKT1, AKT serine/threonine kinase 1; 3'UTR, 3'-untranslated region; Wt, wild-type; mut, mutant; NC, negative control. 

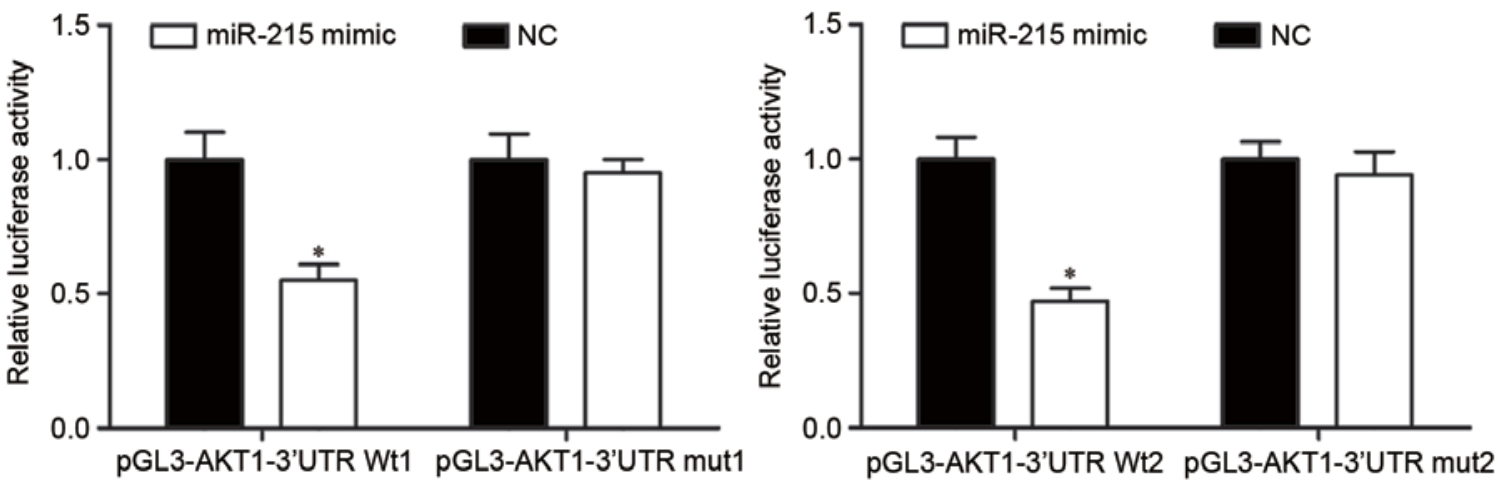

Figure 4. Relative luciferase activities measured in HEK293T cells following co-transfection with the luciferase report vectors and miR-215 mimic or NC ${ }^{*} \mathrm{P}<0.05$ compared with NC-transfected cells. miR-215, microRNA-215; NC, negative control; AKT1, AKT serine/threonine kinase 1; 3'UTR, 3'-untranslated region; Wt, wild-type; mut, mutant.

MDA-MB-231 cells compared with cells transfected with $\mathrm{NC}(\mathrm{P}<0.05$; Fig. 3B).

AKT1 is a direct target of miR-215. To determine whether miR-215 could directly target AKT1, a luciferase reporter assay was performed. Luciferase reporter vectors with the miR-215 mimic or NC were introduced into HEK293T cells. miR-215-transfected HEK293T cells further transfected with the pGL3-AKT1-3'UTR Wt1 and Wt2 luciferase report vectors exhibited a significant decrease in luciferase activity compared with NC-transfected cells with the reporter vectors (P<0.05; Fig. 4). By contrast, pGL3-AKT1-3'UTR Mut1 and Mut2 luciferase reporter vectors abrogated the repression of luciferase activity associated with miR-215 overexpression in HEK293T cells. These results suggested that AKT1 was a direct target of miR-215.

AKT1 promotes the proliferation and invasion of breast cancer cells. The results of the present study had demonstrated that miR-215 overexpression inhibited the proliferative and invasive capacity of breast cancer cells, and AKT1 had been implicated as a direct target of miR-215. Therefore the association of AKT1 with the proliferation and invasion of breast cancer cells was investigated. AKT1 siRNA was used to decrease AKT1 expression. Following transfection, western blot analysis demonstrated that AKT1 protein expression was decreased in the AKT1 siRNA groups compared with in the scrambled siRNA groups ( $\mathrm{P}<0.05$; Fig. 5A).

An MTT assay revealed that AKT1 siRNA transfection inhibited the proliferation of MCF-7A and MDA-MB-231 cells compared with transfection with scrambled siRNA $(\mathrm{P}<0.05$; Fig. 5B). In addition, AKT1 siRNA decreased the invasive capacity of MCF-7A and MDA-MB-231 cells compared with the scrambled siRNA groups $(\mathrm{P}<0.05 ;$ Fig. $5 \mathrm{C})$. These data indicated that the reduced expression of AKT1 inhibited cell proliferation and invasion in a similar manner to miR-215 overexpression in MCF-7A and MDA-MB-231 cells, which suggested that AKT1 was the functional target of miR-215 in breast cancer.

\section{Discussion}

In the present study, it was identified that miR-215 was significantly downregulated in breast cancer tissues and cell lines compared with adjacent non-tumor breast tissues and a normal human breast epithelial cell line, respectively. miR-215 overexpression inhibited the proliferation and invasion of breast cancer cells. In addition, AKT1 was identified as a novel target of miR-215; therefore, it was hypothesized that miR-215 acted as a tumor suppressor through the downregulation of AKT1 in breast cancer cells. The results suggested that miR-215 should be investigated as a potential therapeutic agent in the treatment of breast cancer.

Previous studies identified that miR-215 was upregulated in gastric (26-28) and cervical (29) cancer. The expression level of miR-215 was demonstrated to be associated with the International Federation of Gynecology and Obstetrics stage, the histological grade, and the extents of vascular invasion and lymph node metastasis of cervical cancer; the 5-year survival rate was lower in patients with stage II cervical cancer who exhibited miR-215 overexpression (29). However, previous studies have also demonstrated that miR-215 may be downregulated in epithelial ovarian (30), pancreatic (31), non-small cell lung (32), breast (33) and colon (34) cancer. In the study on pancreatic cancer, a low expression level of miR-215 was significantly associated with a large tumor size, an advanced TNM stage, a high extent of lymph node metastasis and vessel invasion, and lower overall survival time; multivariate regression analysis demonstrated that miR-215 underexpression was an independent unfavorable prognostic factor for patients (31). miR-215 expression was also reported to be associated with a higher tumor grade, human epidermal growth factor receptor 2 (HER2)-positivity, HER2-positive breast cancer subtype and lymph node metastasis in breast cancer (33). These studies have indicated that miR-215 may be a diagnostic and prognostic biomarker for a number of types of cancer.

In certain types of cancer, miR-215 has been identified as a tumor suppressor. For example, in epithelial ovarian cancer, ectopic miR-215 expression suppressed cell proliferation, promoted apoptosis and increased sensitivity to chemotherapy drugs (30). Ge et al (35) reported that miR-215 overexpression inhibited cell proliferation, promoted apoptosis and increased sensitivity to chemotherapy drugs in ovarian cancer cells. Hou et al (32) also demonstrated that the restoration of the expression of miR-215 inhibited proliferation and invasion and promoted apoptosis in vitro, and suppressed tumorigenicity and metastasis in vivo, in non-small cell lung cancer cells. 

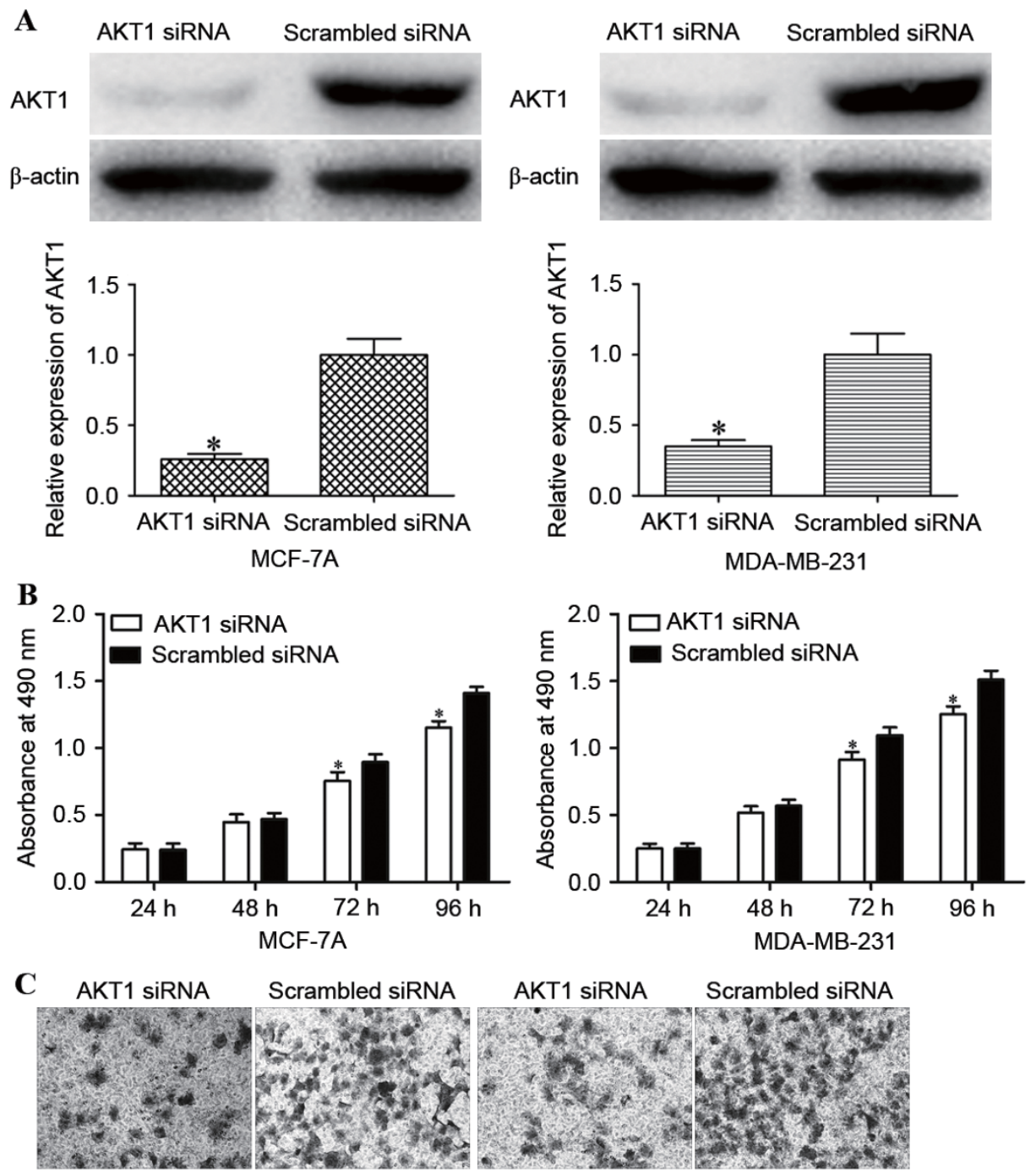

AKT1 siRNA Scrambled siRNA
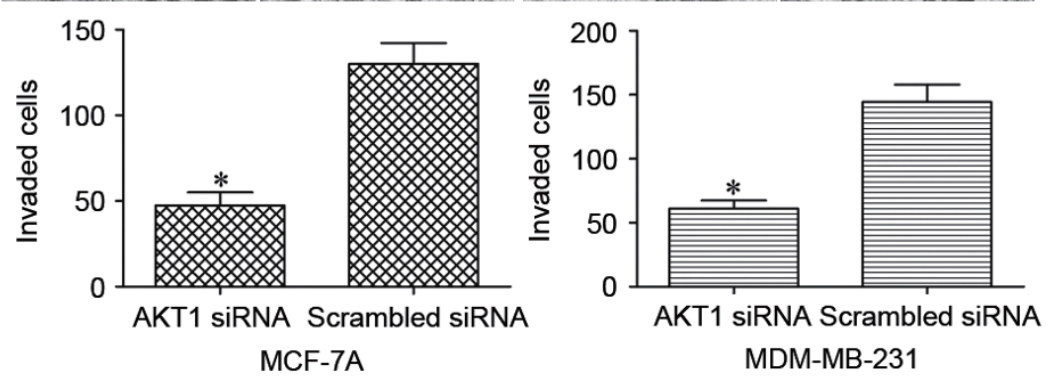

Figure 5. Effects of decreased AKT1 expression on the proliferation and invasion of breast cancer cells. (A) Western blot analysis revealed that AKT1 was markedly downregulated in MCF-7A and MDA-MB-231 cells transfected with AKT1 siRNA. (B) MCF-7A and MDA-MB-231 cells transfected with AKT1 siRNA exhibited a decreased rate of proliferation compared with cells transfected with scrambled siRNA. (C) Underexpression of AKT1 decreased the relative invasive capacity of MCF-7A and MDA-MB-231 cells. "P<0.05 compared with scrambled siRNA-transfected cells. AKT1, AKT serine/threonine kinase 1; siRNA, small interfering RNA.

However, in gastric cancer and cervical cancer, miR-215 has previously been demonstrated to act as an oncogene. Previous studies demonstrated that miR-215 enhanced the proliferation, migration, invasion and metastasis of gastric cancer cells (26-28). These previous studies may appear contradictory, in that miR-215 acted as an oncogene in certain types of cancer, and a tumor suppressor in others. This contradiction may be explained by the 'imperfect complementarity' of the interactions between miRNAs and target genes (36).

miRNAs perform critical functions in a variety of cellular processes by binding to the $3^{\prime}$ UTR of target mRNAs $(10,11)$.
Several targets of miR-215 have been identified, including runt-related transcription factor 1 (28) and RB transcriptional corepressor 1 (26) in gastric cancer, X-linked inhibitor of apoptosis in epithelial ovarian cancer (30), and zinc finger E-box binding homeobox 2 in pancreatic cancer (31) and non-small cell lung cancer (32). In our study, AKT1 was validated as a novel target of miR-215 through four experiments. Firstly, TargetScan online software and miRanda predicted that the AKT1 3'UTR contained two miR-215 seed matches. Secondly, western blot analysis results demonstrated that miR-215 mimic decreased the AKT1 expression level of breast cancer cells. 
Thirdly, a luciferase report assay revealed that miR-215 targeted the AKT1 3'UTR. Finally, the consequences of reducing AKT1 expression were similar to miR-215 overexpression in breast cancer cells. These results have prompted us to hypothesize that AKT1 is a direct target of miR-215, and that miR-215 produces its anti-tumor effects in breast cancer cells through the downregulation of AKT1.

AKT1 is a highly conserved serine/threonine kinase and a crucial factor of the PI3K/AKT pathway, which regulates various cellular processes, including proliferation, apoptosis, migration, invasion and metabolism $(37,38)$. AKT1 is one of the most frequently activated kinases in human cancer $(39,40)$. It has been demonstrated that AKT1 is upregulated in a number of types of cancer, including prostate and breast cancer, and ovarian carcinomas (41). Therefore, AKT1 is considered a valuable therapeutic target for several types of cancer. As it has been identified that miR-215 targeted AKT1 to inhibit breast cancer cell proliferation and invasion, miR-215/AKT1-based therapy may be a promising treatment for breast cancer.

In conclusion, the present study has demonstrated that the level of miR-215 expression was decreased in breast cancer, and that miR-215 overexpression inhibited the proliferation and invasion of breast cancer cells by targeting AKT1. The present study has provided novel insight into the molecular mechanisms underlying the proliferation and metastasis of breast cancer. However, as the regulatory role of miR-215 in the rapid proliferation and metastasis of breast cancer may be complex, the topic requires further investigation in further studies.

\section{References}

1. Singh R, Yadav V, Kumar S and Saini N: MicroRNA-195 inhibits proliferation, invasion and metastasis in breast cancer cells by targeting FASN, HMGCR, ACACA and CYP27B1. Sci Rep 5: 17454, 2015.

2. Siegel RL, Miller KD and Jemal A: Cancer statistics, 2015. CA Cancer J Clin 65: 5-29, 2015.

3. Eroles P, Tormo E, Pineda B, Espin E and Lluch A: MicroRNAs in breast cancer: One more turn in regulation. Curr Drug Targets 17: 1083-1100, 2016.

4. Nakamura S, Yagata $H$, Ohno S, Yamaguchi $H$, Iwata $H$, Tsunoda N, Ito Y, Tokudome N, Toi M, Kuroi K and Suzuki E: Multi-center study evaluating circulating tumor cells as a surrogate for response to treatment and overall survival in metastatic breast cancer. Breast Cancer 17: 199-204, 2010.

5. Gong Y, He T, Yang L, Yang G, Chen Y and Zhang X: The role of miR-100 in regulating apoptosis of breast cancer cells. Sci Rep 5: $11650,2015$.

6. Muenst S, Däster S, Obermann EC, Droeser RA, Weber WP, von Holzen U, Gao F, Viehl C, Oertli D and Soysal SD: Nuclear expression of snail is an independent negative prognostic factor in human breast cancer. Dis Markers 35: 337-344, 2013.

7. Lu L, Ju F, Zhao H and Ma X: MicroRNA-134 modulates resistance to doxorubicin in human breast cancer cells by downregulating $\mathrm{ABCC1}$. Biotechnol Lett 37: 2387-2394, 2015.

8. Zielinska HA, Bahl A, Holly JM and Perks CM: Epithelialto-mesenchymal transition in breast cancer: A role for insulin-like growth factor I and insulin-like growth factor-binding protein 3 ? Breast Cancer (Dove Med Press) 7: 9-19, 2015.

9. Monroig Pdel C, Chen L, Zhang S and Calin GA: Small molecule compounds targeting miRNAs for cancer therapy. Adv Drug Deliv Rev 81: 104-116, 2015.

10. Bartel DP: MicroRNAs: Target recognition and regulatory functions. Cell 136: 215-233, 2009.

11. Smith L, Baxter EW, Chambers PA, Green CA, Hanby AM, Hughes TA, Nash CE, Millican-Slater RA, Stead LF, Verghese ET and Speirs V: Down-regulation of miR-92 in breast epithelial cells and in normal but not tumour fibroblasts contributes to breast carcinogenesis. PLoS One 10: e0139698, 2015.
12. Fei B and $\mathrm{Wu} \mathrm{H}: \mathrm{MiR}-378$ inhibits progression of human gastric cancer MGC- 803 cells by targeting MAPK 1 in vitro. Oncol Res 20: 557-564, 2012.

13. Wang Z, Yin B, Wang B, Ma Z, Liu W and Lv G: MicroRNA-210 promotes proliferation and invasion of peripheral nerve sheath tumor cells targeting EFNA3. Oncol Res 21: 145-154, 2013.

14. Misawa A, Katayama R, Koike S, Tomida A, Watanabe T and Fujita N: AP-1-Dependent miR-21 expression contributes to chemoresistance in cancer stem cell-like SP cells. Oncol Res 19: 23-33, 2010.

15. OhdairaH,Sekiguchi M,Miyata K and Yoshida K: MicroRNA-494 suppresses cell proliferation and induces senescence in A549 lung cancer cells. Cell Prolif 45: 32-38, 2012.

16. Cao Q, Liu F, Ji K, Liu N, He Y, Zhang W and Wang L: MicroRNA-381 inhibits the metastasis of gastric cancer by targeting TMEM16A expression. J Exp Clin Cancer Res 36: 29, 2017.

17. Tomasetti M, Santarelli L, Neuzil J and Dong L: MicroRNA regulation of cancer metabolism: Role in tumour suppression. Mitochondrion 19: 29-38, 2014

18. Rusek AM, Abba M, Eljaszewicz A, Moniuszko M, Niklinski J and Allgayer H: MicroRNA modulators of epigenetic regulation, the tumor microenvironment and the immune system in lung cancer. Mol Cancer 14: 34, 2015.

19. Hurst DR, Edmonds MD and Welch DR: Metastamir: The field of metastasis-regulatory microRNA is spreading. Cancer Res 69: 7495-7498, 2009.

20. Tang H, Liu P, Yang L, Xie X, Ye F, Wu M, Liu X, Chen B, Zhang L and Xie X: miR-185 suppresses tumor proliferation by directly targeting E2F6 and DNMT1 and indirectly upregulating BRCA1 in triple-negative breast cancer. Mol Cancer Ther 13: 3185-3197, 2014.

21. Pu Q, Huang Y, Lu Y, Peng Y, Zhang J, Feng G, Wang C, Liu L and Dai Y: Tissue-specific and plasma microRNA profiles could be promising biomarkers of histological classification and TNM stage in non-small cell lung cancer. Thorac Cancer 7: 348-354, 2016.

22. Song J, Bai Z, Zhang J, Meng H, Cai J, Deng W, Bi J, Ma X and Zhang Z: Serum microRNA-21 levels are related to tumor size in gastric cancer patients but cannot predict prognosis. Oncol Lett 6: 1733-1737, 2013.

23. Bartels CL and Tsongalis GJ: MicroRNAs: Novel biomarkers for human cancer. Ann Biol Clin (Paris) 68: 263-272, 2010.

24. Livak KJ and Schmittgen TD: Analysis of relative gene expression data using real-time quantitative PCR and the 2(-Delta Delta C(T)) method. Methods 25: 402-408, 2001.

25. Ju X, Katiyar S, Wang C, Liu M, Jiao X, Li S, Zhou J, Turner J, Lisanti MP, Russell RG, et al: Aktl governs breast cancer progression in vivo. Proc Natl Acad Sci USA 104: 7438-7443, 2007.

26. Deng Y, Huang Z, Xu Y, Jin J, Zhuo W, Zhang C, Zhang X, Shen M, Yan X, Wang L, et al: MiR-215 modulates gastric cancer cell proliferation by targeting RB1. Cancer Lett 342: 27-35, 2014.

27. Xu YJ and Fan Y: MiR-215/192 participates in gastric cancer progression. Clin Transl Oncol 17: 34-40, 2015.

28. Li N, Zhang QY, Zou JL, Li ZW, Tian TT, Dong B, Liu XJ, Ge S, Zhu Y, Gao J and Shen L: miR-215 promotes malignant progression of gastric cancer by targeting RUNX1. Oncotarget 26: 4817-4828, 2016.

29. Liang H, Li Y, Luo RY and Shen FJ: MicroRNA-215 is a potential prognostic marker for cervical cancer. J Huazhong Univ Sci Technolog Med Sci 34: 207-212, 2014.

30. Ge G, Zhang W, Niu L, Yan Y, Ren Y and Zou Y: miR-215 functions as a tumor suppressor in epithelial ovarian cancer through regulation of the $\mathrm{X}$-chromosome-linked inhibitor of apoptosis. Oncol Rep 35: 1816-1822, 2016.

31. Li QW, Zhou T, Wang F, Jiang M, Liu CB, Zhang KR, Zhou Q, Tian Z and $\mathrm{Hu}$ KW: MicroRNA-215 functions as a tumor suppressor and directly targets ZEB2 in human pancreatic cancer. Genet Mol Res 14: 16133-16145, 2015.

32. Hou Y, Zhen J, Xu X, Zhen K, Zhu B, Pan R and Zhao C: miR-215 functions as a tumor suppressor and directly targets ZEB2 in human non-small cell lung cancer. Oncol Lett 10: 1985-1992, 2015.

33. Zhou SW, Su BB, Zhou Y, Feng YQ, Guo Y, Wang YX, Qi P and $\mathrm{Xu} \mathrm{S}$ : Aberrant miR-215 expression is associated with clinical outcome in breast cancer patients. Med Oncol 31: 259, 2014.

34. Karaayvaz M, Pal T, Song B, Zhang C, Georgakopoulos P, Mehmood S, Burke S, Shroyer K and Ju J: Prognostic significance of miR-215 in colon cancer. Clin Colorectal Cancer 10: 340-347, 2011. 
35. Ge G, Zhang W, Niu L, Yan Y, Ren Y and Zou Y: miR-215 functions as a tumor suppressor in epithelial ovarian cancer through regulation of the $\mathrm{X}$-chromosome-linked inhibitor of apoptosis. Oncol Rep 35: 1816-1822, 2016.

36. Yu Z, Ni L, Chen D, Zhang Q, Su Z, Wang Y, Yu W, Wu X, Ye J, Yang $\mathrm{S}$, et al: Identification of miR-7 as an oncogene in renal cell carcinoma. J Mol Histol 44: 669-677, 2013.

37. Agarwal E, Brattain MG and Chowdhury S: Cell survival and metastasis regulation by Akt signaling in colorectal cancer. Cell Signal 25: 1711-1719, 2013.

38. Kang B, Hao C, Wang H, Zhang J, Xing R, Shao J, Li W, $\mathrm{Xu} \mathrm{N}$, Lu Y and Liu S: Evaluation of hepatic-metastasis risk of colorectal cancer upon the protein signature of PI3K/AKT pathway. J Proteome Res 7: 3507-3515, 2008.
39. Altomare DA and Testa JR: Perturbations of the AKT signaling pathway in human cancer. Oncogene 24: 7455-7464, 2005.

40. Zhang G, Liu Z, Xu H and Yang Q: miR-409-3p suppresses breast cancer cell growth and invasion by targeting Akt1. Biochem Biophys Res Commun 469: 189-195, 2016.

41. Sun M, Wang G, Paciga JE, Feldman RI, Yuan ZQ, Ma XL, Shelley SA, Jove R, Tsichlis PN, Nicosia SV and Cheng JQ: AKT1/PKBalpha kinase is frequently elevated in human cancers and its constitutive activation is required for oncogenic transformation in NIH3T3 cells. Am J Pathol 159: 431-437, 2001. 